DOI: https://doi.org/10.15407/techned2018.05 $=\underline{044}$

\title{
CURRENT STATE AND DEVELOPMENT TRENDS OF AC VOLTAGE CONVERTERS WITH TRANSFORMER-AND-SWITCHES EXECUTIVE STRUCTURE
}

Journal

Publisher

ISSN

Issue

Pages
Tekhnichna elektrodynamika

Institute of Electrodynamics National Academy of Science of Ukraine 1607-7970 (print), 2218-1903 (online)

No 5, 2018 (September/October)

$44-51$

\section{Authors}

K.O. Lypkivskyi ${ }^{\star}$, A.G. Mozharovskyi ${ }^{\star *}$

Institute of Electrodynamics National Academy of Sciences of Ukraine,

pr. Peremohy, 56, Kyiv, 03057, Ukraine,

e-mail: lypkivskyk@ukr.net; AnatMozhrvsk@ukr.net

* ORCID ID : http://orcid.org/0000-0002-3292-1360

** ORCID ID : http://orcid.org/0000-0001-9801-2728

AC voltage converters (regulators, stabilizers) with TSES - transformer-and-switches executive structure are distinguished by high energy indices and electromagnetic compatibility with the supply network and the consumer. In the article, characteristic objects of research and patenting of devices of this class are analyzed which, due to a priori multivariance, can differ substantially in the type of construction of the control units and their integration, the law of sectioning of the windings of the transforming element. The effectiveness and expediency of strict adherence to the already approved basic provisions of the TSES theory is substantiated and the ways of their further improvement are defined with the expansion of the circle of significant quality indicators of voltage converters on their basis. References 24. 
Key words: transformer-and-switches executive, AC voltage converter, sectionalized winding, tap changing transformer, discrete smart transformer, voltage stabilizer, regulator.

Received: 05.03.2018

Accepted: 03.05.2018

Published: 16.08.2018

\section{References}

1. Bimal K. Bose. Power Electronics - Why the Field is so Exciting? IEEE Power Electronics Society Newsletter . Fourth

Quarter. 2007. Vol. 19. No 4. Pp. 11-20.

2. Ram G., Prasanth V., Bauer P., Barthlein E.M. Comparative analysis of on-load tap changing (OLTC) transformer topologies. Power Electronics and Motion Control Conference and Exposition (PEMC). 2014 16-th $^{\text {th }}$ International. IEEE. 21-24 Sept. 2014. Antalya, Turkey. Pp. 918-923. DOI:

http://dx.doi.org/10.1109/EPEPEMC.2014.6980624

3. Electronic Tap Switching Voltage Regulator. Available at: http://www.ustpower.com/compari ng-automatic-voltage-regulation-technologies/avr-guide-electronic-tap-switching-voltage-regula tor/ (accessed 01.03.2018).

4. Willems W., Vandoorn T.L., De Kooning J.D., Vandevelde L. Development of a smart transformer to control the power exchange of a microgrid. 4-th International Conference Innovative Smart Grid Technologies Conference

Europe (ISGT - Europe 2013). IEEE. 6-9 Oct. 2013. At Lyngby. Denmark. Pp. 1-5. DOI: http://dx.doi.org/ 10.1109/ISGTEurope.2013.6695300

5. Lypkivskyi K.O. Transformer-and-switches executive structures of alternating current voltage converters. Kyiv: Naukova Dumka, 1983. 216 p. (Rus)

6. William O. Kramer, Alireza Daneshpooy. Static voltage regulator and controller. US Patent 6351106B1. Feb. 26, 2002.

7. Robert C. Degeneff, Steven Raedy. Regulator with asymmetrical voltage increase/decrease capability for utility system. US Patent 5990667. Nov. 23, 1999.

8. James W. Kronberg. Digitally-controlled AC voltage stabilizer. US Patent $6417651 B 1$. 
09.07.2002.

9. Liu Zhiyong. Voltage deviation adjustor. Patent CN103178527B. 2015.07.01.

10. Mohammad Sarvi, Haniyeh Marefatjou. Compensation of Voltage Single-Phase SAG and SWELL Using Dynamic Voltage Restorer and Difference Per-Unit Value Method. International Journal of Electrical and Computer Engineering

(IJECE). Vol. 3. No 1. February 2013. Pp. 83-92. DOI:

https://doi.org/10.11591/ijece.v3i1.2134

11. Santilio F.P., Silva T.V., Oliveira J.C., Barbosa J.A.F. A computational and experimental performance analysis of an electromagnetic voltage regulator proposal throughout controlled series voltage injection. Renewable Energies and Power Quality Journal (RE\&PQJ). Vol. 1. No 10. April 2012. Pp. 767-772. DOI:

https://doi.org/10.24084/repqj10.465

12. Lypkivskyi K.O. Duality of construction of transformer-and-switches executive structures of discrete stabilizers and AC voltage regulators. Tekhnichna Elektrodynamika. 2010. No 6. Pp. 16-23. (Ukr)

13. 5KVA Voltage Stabilizer Circuit. Available at: https://makingcircuits.com/blog/5kva-voltagestabilizer-circuit/

(accessed 01.03.2018).

14. Paul D. Brooks. Tap switching power supply apparatus and methods for supporting multiple input voltages. US Patent 9568931B1. Feb.14, 2017.

15. Tonkal V.E., Lypkivskyi K.O. Alternating voltage regulators and stabilizers whit undistorted out-put voltage. Electrical energy supply and other application. $3^{\text {rd }}$ Power electronics conference Budapest'77.

1977. Vol. 6.15. Pp. 1-15.

16. Shvarts D.L., Shevelev V.O., Gendelman G.A., Antonov Yu.G., Golberg G.Yu. Transformer with stepped voltage regulation. Patent UA No 5107. 28.12.94. (Ukr)

17. Lypkivskyi K.O., Tonkal V.E., Ozerianskyi A.O. Device for regulation of alternating voltage. Patent USSR No 413468. 30.01.1974. (Rus)

18. Robert C. Degeneff, Friedrich K. Schaeffer; Robert H. Frazer, David A. Torrey, Osman Demirci. Tap changing system having discrete cycle modulation and fauld rotation for coupling to an inductive device. US Patent 5604423. Feb. 18. 1997.

19. Lypkivskyi K.O. Systematization of transformer-and-switches executive structures of voltage converters with switches elements in the field of power current. Tekhnichna Elektrodynamika 2011.

No 3. Pp. 26-32. (Ukr)

20. Steven M. Raedy. 3-Phase electronic tap changer commutation and device. US Patent 7737667B2. Jun. 15. 2010.

21. Korotetskyi Yu.L. Device for regulation of alternating voltage. Patent UA No 36418.

15.07.2003. (Ukr)

22. Shesterenko V.Ye., Shesterenko O.V. The method of discrete voltage regulation by booster transformers. Patent UA No 72050. 10.08.2012. (Ukr)

23. Allan David Crane, Warren Mark Blewitt. Power converters. Patent US 2015/0295498A1. Oct. 15, 2015.

24. Lypkivskyi K.O., Mozharovskyi A.G. Simulation of the transformative elements with 
sectioning of the win-dings as part of AC voltage source converters. Tekhnichna Elektrodynamika 2016.

No 3. Pp. 39-44. (Ukr)

DOI: https://doi.org/10.15407/techned2016.03.039

\section{PDF}

